\title{
Some Applications for Series Impedance Elements in Radio Frequency Immittance Measurements
}

\author{
Leslie E. Huntley* \\ Institute for Basic Standards, National Bureau of Standards, Boulder, Colorado 80302
}

(January 29, 1970)

\begin{abstract}
A series impedance element equipped with coaxial connectors may be evaluated as a two-port network. Precision connectors greatly reduce the uncertainties associated with the series connection, making practical several measurement techniques which involve series impedances. This paper discusses techniques for extending the range of immittance bridges to high values of admittance or impedance, for measuring very small admittances with incremental standards of ordinary range, and for using a bridge to measure its own reference open-circuit admittance or short-circuit impedance.
\end{abstract}

Key words: Immittance measurement; radio frequency; series elements; two-port standards.

\section{Introduction}

A number of measuring techniques which are commonly used at audio frequencies, have long been considered impractical for use at radio frequencies. Among these are the techniques which require connecting one component in series with another. The use of precision coaxial connectors $(1,2)$, greatly reduces uncertainties in series and shunt immittances associated with the connection, making it practical to use series connected components for precision measurements at radio frequencies.

When a circuit element is equipped with precision connectors, the elements of its equivalent circuit are fixed by the shielding afforded by the coaxial construction and by the precisely repeatable connection. A shielded series element may be treated as a two-port network and represented by an equivalent pi or tee at a given frequency, and if it is equipped with precision connectors, the elements of the equivalent pi or tee may be accurately evaluated by open- and shortcircuit measurements. This paper discusses the use of series immittances in the form of precision two-port standards in extending the range of immittance measuring instruments to large immittances, in measuring low-valued admittances in terms of relatively large incremental standards, and in measuring the impedance of the reference short-circuit of an impedance bridge, or the admittance of the reference open-circuit of an admittance bridge.

*Electromagnetics Division, Circuit Standards Section, National Bureau of Standards, Boulder Laboratories, Boulder, Colo. 80302.

\section{Method for Evaluating Two-Port Immittances}

A two-port immittance may be represented by an equivalent pi network such as that of figure 1, at any single frequency [3]. The procedure for measuring the elements of the pi is well known, but is reviewed here for the sake of convenience.

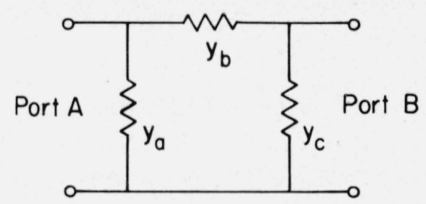

FIGURE 1. Pi equivalent of two-port network.

With port A of the two-port connected to an admittance bridge, and port B shorted, the input admittance measured by the bridge is

$$
Y_{1}=Y_{a}+Y_{b} .
$$

When the short is removed, port B is open circuited, and the bridge measures the input admittance

$$
Y_{2}=Y_{a}+\frac{Y_{b} Y_{c}}{Y_{b}+Y_{c}} .
$$

With port B connected to the bridge and port A shorted, the input admittance is given by

$$
Y_{3}=Y_{b}+Y_{c} .
$$


When the short is removed, port $\mathrm{A}$ is open, and the input admittance is

$$
Y_{4}=Y_{c}+\frac{Y_{a} Y_{b}}{Y_{a}+Y_{b}}
$$

Note that the two-port must be equipped with nominally identical connectors for this series of measurements to be possible. Equations (1), (2), and (3) are solved simultaneously for $Y_{a}, Y_{b}$, and $Y_{c}$, yielding

$$
\begin{aligned}
& Y_{b}=\left[\left(Y_{1}-Y_{2}\right) Y_{3}\right]^{1 / 2}, \\
& Y_{a}=Y_{1}-Y_{b},
\end{aligned}
$$

and

$$
Y_{c}=Y_{3}-Y_{b} \text {. }
$$

So far it has been assumed that the open circuit actually represents zero admittance across the open port, and that the short circuit actually represents zero impedance. This assumption is not accurate. The short may have an impedance consisting of about $0.5 \mathrm{~m} \Omega$ (milliohm) resistance and $5 \times 10^{-12}$ henry inductance, and the open may have an admittance consisting of a fringing capacitance of the order of $0.2 \mathrm{pF}$ [4]. The conductance of an open circuit can usually be neglected, as can the error caused by a nonzero short where all admittances are of the order of 0.1 mho or smaller. When the fringing capacitance at the open end of the two-port is taken into account, (2) becomes

$$
Y_{2}=Y_{a}+\frac{Y_{b}\left(Y_{c}+Y_{f 1}\right)}{Y_{b}+Y_{c}+Y_{f 1}}
$$

and (4) becomes

$$
Y_{4}=Y_{c}+\frac{Y_{b}\left(Y_{a}+Y_{f 2}\right)}{Y_{a}+Y_{b}+Y_{f 2}},
$$

where $Y_{f 1}$ is the "fringe admittance" at one open end of the two-port, and $Y_{f 2}$ is the fringe admittance at the other. Fringe admittance is used instead of fringe capacitance because the equations than apply to the more general case of a lossy open circuit. If the twoport is equipped with precision connectors, the two fringe admittances will be very nearly equal.

The fringe admittance at the bridge terminal, $Y_{f 3}$, is removed when connection is made to the bridge terminal. Each of the measurements (1) through (4) is therefore in error by the amount $Y_{f 3}$, and this admittance must be added to the measured admittance in order to obtain the correct values for $Y_{a}+Y_{b}$, etc.

Note that $Y_{f 3}$ will be different from $Y_{f 1}$ and $Y_{f 2}$ unless the connectors are sexless. Even when precision connectors, which are sexless by definition, are used, $Y_{f 3}$ may be different from $Y_{f 1}$ and $Y_{f 2}$ because of differences in the configuration of conductors near the open circuit. For example, the fringe capacitance of a connector mounted flush with a large ground plane will differ considerably from that of an identical connector at the end of a two-port several inches above the ground plane. The three fringe admittances for precision connectors can be made virtually identical by using a shielding cap instead of an open terminal to provide the reference open circuit admittance. When the corrections for the fringe admittances are made, (1) through (4) must be rewritten

$$
\begin{gathered}
Y_{1}+Y_{f 3}=Y_{a}+Y_{b}, \\
Y_{2}+Y_{f 3}=Y_{a}+\frac{Y_{b}\left(Y_{c}+Y_{f 1}\right)}{Y_{b}+Y_{c}+Y_{f 1}} . \\
Y_{3}+Y_{f 3}=Y_{b}+Y_{c}
\end{gathered}
$$

and

$$
Y_{4}+Y_{f 3}=Y_{c}+\frac{Y_{b}\left(Y_{a}+Y_{f 2}\right)}{Y_{a}+Y_{b}+Y_{f 2}} .
$$

where $Y_{1}, Y_{2}$, etc., still refer to the admittances measured at the bridge terminal. The first three equations of this set may be solved as before, yielding

$Y_{b} \equiv G_{b}+j w C_{b}=\left[\left(Y_{1}-Y_{2}\right)\left(Y_{3}+Y_{f 1}+Y_{f 3}\right)\right]^{1 / 2}$,

and

$$
Y_{a}=Y_{1}+Y_{f 3}-Y_{b},
$$

$$
Y_{c}=Y_{3}+Y_{f 3}-Y_{b} .
$$

Section 3.3 of this paper describes a technique for measuring the reference, or "fringe," admittance of an admittance bridge and of a two-port admittance.

A two-port immittance may also be represented by the equivalent tee of figure 2 , and the elements

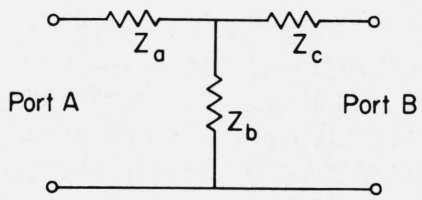

Figure 2. Tee Equivalent of two-port network.

evaluated by measurements with an impedance bridge. With port A connected to the bridge and B open, the bridge measures

$$
Z_{1}+Z_{s 3}=Z_{a}+Z_{b},
$$

where $Z_{s 3}$ is the impedance of the reference short circuit which is removed when the two-port is connected to the bridge, and the effect of fringing capacitance is considered negligible. When port B is shorted, the bridge measures

$$
Z_{2}+Z_{s 3}=Z_{a}+\frac{Z_{b}\left(Z_{c}+Z_{s 1}\right)}{Z_{b}+Z_{c}+Z_{s 1}},
$$

where $Z_{s 1}$ is the impedance of the short on port $\mathrm{B}$, and is not necessarily equal to $Z_{s 3}$. With port $\mathrm{B}$ connected to the bridge and port A open, the bridge measures

$$
Z_{3}+Z_{s 3}=Z_{b}+Z_{c} .
$$


With port A shorted, the bridge measures

$$
Z_{4}+Z_{s 3}=Z_{c}+\frac{Z_{b}\left(Z_{a}+Z_{s 2}\right)}{Z_{a}+Z_{b}+Z_{s 2}}
$$

The first three equations of this set may be solved for $Z_{a}, Z_{b}$, and $Z_{c}$, yielding

$$
\begin{gathered}
Z_{b}=\left[\left(Z_{1}-Z_{2}\right)\left(Z_{3}+Z_{s 1}+Z_{s 3}\right)\right]^{1 / 2}, \\
Z_{a}=Z_{1}+Z_{s 3}-Z_{b},
\end{gathered}
$$

and

$$
Z_{c}=Z_{3}+Z_{s 3}-Z_{b}
$$

Section 3.3 presents a technique for measuring the short-circuit impedances.

Solving (14) for $Y_{b}$ involves taking the square root of a complex number. The solutions are

$$
G_{b}^{2}=\frac{A \pm \sqrt{A^{2}+B^{2}}}{2}
$$

and

$$
C_{b}^{2}=\frac{-A \pm \sqrt{A^{2}+B^{2}}}{2 \omega^{2}}
$$

where

$$
A=\left(G_{1}-G_{2}\right) G_{3}-\omega^{2}\left(C_{1}-C_{2}\right)\left(C_{3}+C_{f 1}+C_{f 3}\right),
$$

and

$$
B=\omega\left[\left(G_{1}-G_{2}\right)\left(C_{3}+C_{f 1}+C_{f 3}\right)+G_{3}\left(C_{1}-C_{2}\right)\right] .
$$

Thus, $G_{b}$ and $C_{b}$ are obtainable in terms of measured conductances and capacitances.

The effect of measurement errors on a measurement of this type may be evaluated by the technique of taking the total differential of the quantity measured [5]. The approximate error in $G_{b}$, denoted by $\delta G_{b}$, is given by

$$
\delta G_{b} \simeq \frac{\partial G_{b}}{\partial A} \delta A+\frac{\partial G_{b}}{\partial B} \delta B,
$$

where $\delta A$ and $\delta B$ are small errors in $A$ and $B$ given by

$$
\begin{aligned}
\delta A \simeq \frac{\partial A}{\partial G_{1}} \delta G_{1}+\frac{\partial A}{\partial G_{2}} \delta G_{2}+\ldots+\frac{\partial A}{\partial C_{f 1}} \delta C_{f 1} & \\
& +\frac{\partial A}{\partial C_{f 3}} \delta C_{f 3}
\end{aligned}
$$

and

$$
\delta B \simeq \frac{\partial B}{\partial G_{1}} \delta G_{1}+\ldots+\frac{\partial B}{\partial C_{f 3}} \delta C_{f 3} .
$$

While there is little to be gained by pursuing the algebra further, it is instructive to take a simple example to illustrate the order of magnitude of the errors to be expected in measurements of this type. If a conductance with sufficiently high $q^{2}(q \simeq 200$ for error of 1 in $10^{4}$ ) is measured, the expression for $G_{b}^{2}$ reduces to $G_{b}^{2} \simeq A$. Furthermore, for a high- $q$ conductance

$$
\omega^{2}\left(C_{1}-C_{2}\right)\left(C_{3}+C_{f 1}+C_{f 3}\right) \ll\left(G_{1}-G_{2}\right) G_{3},
$$

and

$$
\delta G_{b} \simeq \frac{1}{2 G_{b}}\left[G_{3}\left(\delta G_{1}-\delta G_{2}\right)+\left(G_{1}-G_{2}\right) \delta G_{3}\right] .
$$

For the case described, $G_{1} \simeq G_{3} \gg G_{2}$, and

$$
\delta G_{b} \simeq\left(1 / 2 G_{b}\right)\left(2 G_{b} \delta G_{1}\right)=\delta G_{1},
$$

and the error in measuring a pure series conductance by this technique is approximately the same as the error in measuring a two-terminal conductance with the same bridge.

\section{Applications}

\subsection{Range Extension to Very Large Immittances}

Hartshorn [6] shows that capacitors too large for direct measurement may be measured by connecting them in series with smaller known capacitors. The capacitance of the series combination is smaller than that of either capacitor and this capacitance may be made to fall within the range of direct measurement. The same principle may be used in measuring any admittance too large for direct measurement with available instruments. The measurement is accomplished by evaluating the elements of the equivalent pi (fig. 1) then connecting the unknown admittance to the two-port and measuring the input admittance of the combination. The value of the unknown admittance is then calculated from the computed elements of the pi and the measured input admittance of the combination.

If the unknown admittance, $Y_{x}$, is connected to port $\mathrm{B}$ and port $\mathrm{A}$ is connected to the bridge, the measured input admittance is given by

$$
Y_{5}=Y_{a}+\frac{Y_{b}\left(Y_{c}+Y_{x}\right)}{Y_{b}+Y_{c}+Y_{x}}-Y_{f 3}
$$

from which is obtained

$$
Y_{x}=\frac{Y_{b}\left(Y_{5}+Y_{f 3}-Y_{a}\right)}{Y_{a}+Y_{b}-Y_{5}-Y_{f 3}}-Y_{c} .
$$

${ }^{2}$ The Quality Factor of an immittance element, denoted by " $q$," is defined to be the ratio of the major immittance component to the minor immittance component. Thus, $q_{1} \equiv \frac{\omega L}{R}, q_{c} \equiv \frac{\omega C}{G}, q_{r} \equiv \frac{R}{\omega L}$, and $q_{s} \equiv \frac{G}{\omega C}$. 
In some cases, the elements of the pi are of no particular interest, and it is desirable to calculate $Y_{x}$ directly from the measured values $Y_{1}, Y_{2}$, and $Y_{3}$. In this case an expression for $Y_{x}$ is obtained by substituting (14), (15), and (16) into (33):

$$
Y_{x}=\frac{\left(Y_{5}-Y_{2}\right)\left(Y_{3}+Y_{f 3}\right)+\left(Y_{1}-Y_{2}\right) Y_{f 1}}{Y_{1}-Y_{5}} .
$$

After expanding (34) or (35) and separating variables, the error in measuring $G_{x}$ and $C_{x}$ may be evaluated, as before, by taking the total differential of the quantity. The operation is straightforward, if somewhat laborious, and will not be pursued further. An idea of the magnitude of errors to be expected in measurements of this sort may be obtained by considering the case of a pure capacitor measured in series with a pure capacitor-that is, all conductances are zero. For this idealized case, (35) becomes

$$
C_{x}=\frac{\left(C_{5}-C_{2}\right)\left(C_{3}+C_{f 3}\right)+\left(C_{1}-C_{2}\right) C_{f 1}}{C_{1}-C_{5}},
$$

and the error in measuring $C_{x}$ is

$$
\begin{gathered}
\delta C_{x}=\frac{1}{C_{1}-C_{5}}\left[\left(C_{f 1}-C_{x}\right) \delta C_{1}-\left(C_{3}+C_{f 1}+C_{f 3}\right) \delta C_{2}\right. \\
+\left(C_{5}-C_{2}\right) \delta C_{3}+\left(C_{3}+C_{x}+C_{f 3}\right) \delta C_{5} \\
\left.+\left(C_{5}-C_{2}\right) \delta C_{f 3}+\left(C_{1}-C_{2}\right) \delta C_{f 1}\right] .
\end{gathered}
$$

The errors in $C_{1}, C_{2}, C_{3}$, and $C_{5}$ are composed of two components; a calibration error and a random error due to imprecision of the measuring capacitor. If $C_{1}$ and $C_{3}$ are approximately equal, the calibration error will be the same for both, and it is proper to consider the sign of the error term in combining the errors. Since $C_{2}$ and $C_{5}$ may be greatly different from $C_{1}$ and $C_{3}$, there is no assurance the calibration errors are in the same direction; and their signs should not be considered in combining the error terms. Figure 3 shows the accuracy which may be expected in series measurement of capacitors between $10 \mathrm{pF}$ and $0.01 \mu \mathrm{F}$ assuming the error in measuring $C_{1}, C_{2}, C_{3}$, and $C_{5}$ to be $\pm(0.05 \%+$ $0.05 \mathrm{pF}$ ), the error in $C_{f 1}$ and $C_{f 3}$ to be about $0.001 \mathrm{pF}$, and

$$
\begin{aligned}
C_{1} & \simeq C_{3} \simeq 1000 \mathrm{pF} \\
C_{2} & \simeq 5 \mathrm{pF} \\
C_{f_{1}} & \simeq C_{f 3} \simeq 0.2 \mathrm{pF},
\end{aligned}
$$

and rms addition of the random errors. The accuracy obtainable by direct measurement under the same conditions is shown for comparison.

The range of admittances which can be measured with a given instrument by this method lies between zero (two-port open) and infinity (two-port shorted). Because the accuracy is not as good as that obtained for direct measurement, only values outside the range of available instruments will ordinarily be measured in this way. The technique was tested by measuring a number of capacitors directly, and in series with a two-port capacitor. The results appear in table 1 . The good agreement between the two methods shows there are no serious errors inherent in the technique.

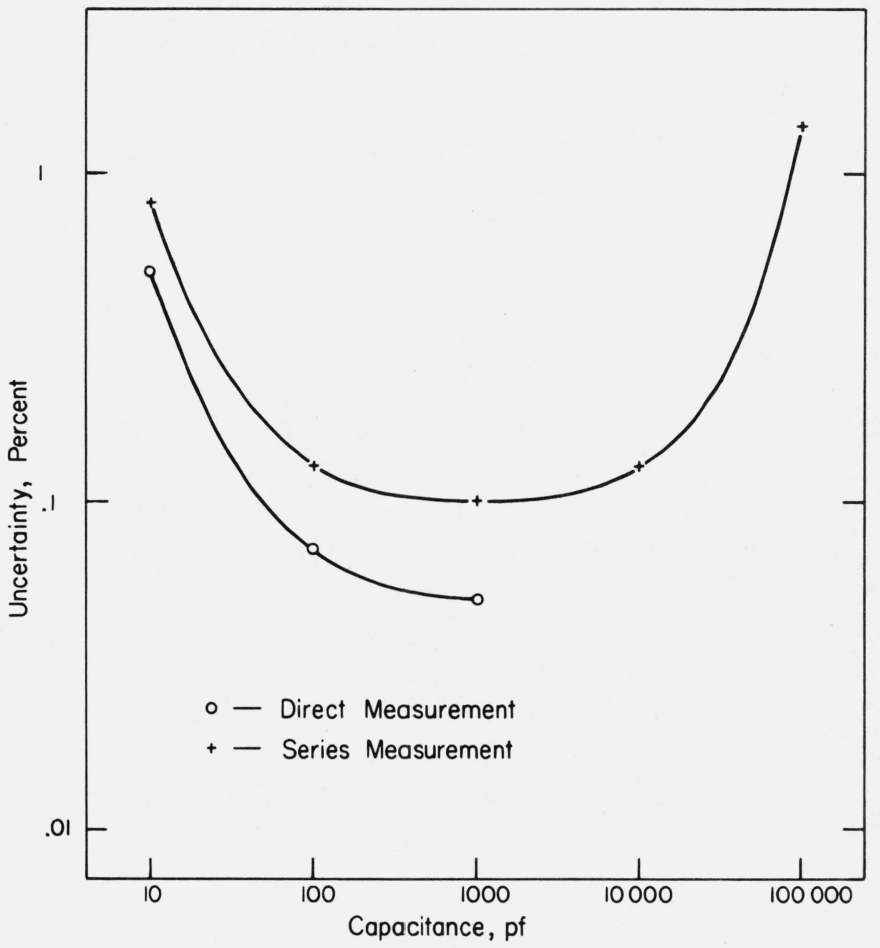

FIGURE 3. Accuracy obtainable in measurement of capacitance by direct measurement, and by series measurement.

TABLE 1. Capacitance measured at $3 \mathrm{MHz}$ by direct measurement and by measurement in series with a 100-pF capacitor

\begin{tabular}{l|c|c|c}
\hline \hline $\begin{array}{c}\text { Nominal } \\
\text { capacitance }\end{array}$ & $\begin{array}{c}\text { Direct } \\
\text { measure- } \\
\text { ment } \\
\mathrm{pF}\end{array}$ & $\begin{array}{c}\text { Series } \\
\text { measure- } \\
\text { ment } \\
\mathrm{pF}\end{array}$ & $\begin{array}{c}\text { Difference } \\
\text { (percent) }\end{array}$ \\
\hline $1000 \mathrm{pF}(\mathrm{A})$ & 1006.77 & 1006.62 & 0.014 \\
$1000 \mathrm{pF}(\mathrm{B})$ & 1002.76 & 1002.63 & .013 \\
$500 \mathrm{pF}$ & 501.78 & 501.71 & .014 \\
$200 \mathrm{pF}$ & 200.66 & 200.71 & -.025 \\
$100 \mathrm{pF}$ & 101.06 & 101.09 & -.03 \\
\hline
\end{tabular}

The usable range of an impedance bridge may be similarly extended by connecting an unknown large impedance in parallel with a smaller impedance whose value is within the range of the bridge. In this case, the two-port is more conveniently represented by the equivalent tee of figure 2 . In practice, the two-port may be formed by connecting the smaller impedance to one port of a coaxial tee junction and using the other two as active ports (fig. 4). In this case, the elements of the tee are obtained by impedance measurements, and the unknown impedance, $Z_{x}$, is given by

$$
Z_{x}=\frac{\left(Z_{5}-Z_{2}\right)\left(Z_{3}+Z_{S 3}\right)+\left(Z_{1}-Z_{2}\right) Z_{S 1}}{Z_{1}-Z_{5}} .
$$


Here again, the range of the bridge is extended to cover impedances between zero (two-port shorted) to infinity (two-port open).

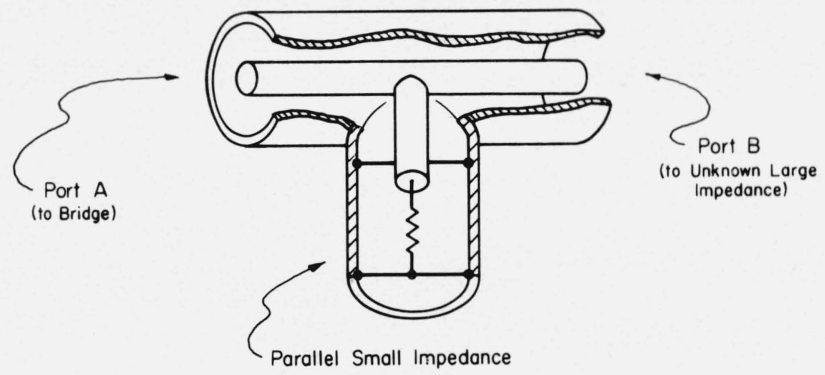

FIGURE 4. Tee arrangement for measuring large impedance in parallel with smaller impedance.

\subsection{Range Extension to Very Small Admittances}

Hartshorn [6] also discusses the measurement of very small capacitors using an incremental capacitor of ordinary range. As he puts it: "Condensers of very low value may also be measured by a series method. A condenser of low value $c$ is placed in series with a variable standard of ordinary range $C$. The capacitance of the combination being $c C /(C+c)$, a small change $\Delta C$ in the standard produces a change in the combination of approximately $\Delta C c^{2} /(C+c)^{2}$, which is for example, 100 times smaller than $\Delta C$ if $C / c=9$. Thus, if the small condenser to be measured is added in parallel with such a combination, and the standard condenser if then diminished until the resultant capacitance is restored to its original value, the capacitance added is equal to the change in the reading of the standard divided by 100 . This method is convenient when no standard of low range is available; but when a standard of the micrometer type is available the substitution method should be employed, as there is always some uncertainty in any estimation of the effect of the connecting lead in a series combinationhowever small the lead may be."

The same principle applies to measuring any small admittance, and the use of precision connectors eliminates much of the "uncertainty in any estimation of the effect of the connecting lead." The series admittance may be represented by an equivalent pi, and evaluated by measurements with an admittance bridge. When the incremental admittance is connected to the two-port, the circuit of figure 5 results.

The input admittance of this network, $Y_{i}$, is

$$
Y_{i}=Y_{a}+\frac{Y_{b}\left(Y_{1}+Y_{c}\right)}{Y_{1}+Y_{b}+Y_{c}}
$$

and the change in input admittance with change in $Y_{1}$ is given by

$$
\Delta Y_{i}=\frac{\left(Y_{1}^{\prime}-Y_{1}\right) Y_{b}^{2}}{\left(Y_{1}+Y_{b}+Y_{c}\right)\left(Y_{1}^{\prime}+Y_{b}+Y_{c}\right)},
$$

where $Y_{1}$ is the initial value, and $Y_{1}^{\prime}$ is the final value. When all the elements of the network are of like kind and are sufficiently pure ( $q$ greater than about 200 for

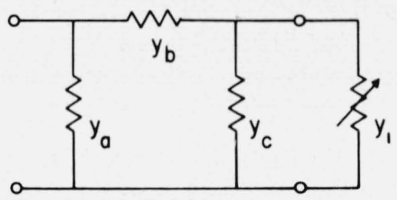

FigURE 5. Equivalent circuit of incremental admittance standard connected to two-port standard for measurement of very small admittances.

1 in $10^{4}$ error) the minor component may be neglected in calculating the change in effective value for the major component.

For sufficiently pure capacitors

$$
\Delta C_{i} \simeq \frac{\Delta C_{1} C_{b}^{2}}{\left(C_{\mathbf{A}}+C_{b}+C_{c}\right)\left(C_{1}^{\prime}+C_{b}+C_{c}\right)},
$$

and for nearly pure conductances

$$
\Delta G_{1} \simeq \frac{\Delta G_{1} G_{b}^{2}}{\left(G_{1}+G_{b}+G_{c}\right)\left(G_{1}^{\prime}+G_{b}+G_{c}\right)} .
$$

Again, some insight into the magnitude of errors to be expected in measurements of this type may be obtained by considering the measurement of a capacitor by an incremental capacitor in series with a two-port capacitor where all capacitors are lossless. The relative error in a measurement of this type, obtained by taking the total differential of (4l) and dividing by $\Delta C_{i}$, is

$$
\begin{aligned}
\frac{\delta\left(\Delta C_{i}\right)}{\Delta C_{i}}=\frac{\delta C_{1}^{\prime}-\delta C_{1}}{C_{1}^{\prime}-C_{1}}- & \frac{\delta C_{1}^{\prime}+\delta C_{b}+\delta C_{c}}{C_{1}^{\prime}+C_{b}+C_{c}} \\
& -\frac{\delta C_{1}+\delta C_{b}+\delta C_{c}}{C_{1}+C_{b}+C_{c}}+\frac{2 \delta C_{b}}{C_{b}} .
\end{aligned}
$$

Perhaps the most useful application of this technique lies in checking the calibration of incremental capacitors against low-valued standards such as those provided by short sections of precision coaxial line. The Field and Sinclair technique [7] may be used for finding corrections proportional to $C^{2}$ in incremental capacitors; and it is easy to show that there are no significant errors proportional to $C^{2}$ or higher-order terms by measuring capacitors of appropriate value over different ranges of the incremental capacitor. None of these techniques are capable of detecting errors proportional to $C$, however, and another technique is needed for a complete calibration of these instruments. 
If the error in measuring the small capacitor is given by

$$
\begin{aligned}
& \delta C_{b}=k C_{b} \pm \epsilon \mathrm{pF} \\
& \delta C_{c}=k C_{c} \pm \epsilon \mathrm{pF} \\
& \delta C_{1}=k C_{1} \pm \epsilon \mathrm{pF} \\
& \delta C_{1}^{\prime}=k C_{1}^{\prime} \pm \epsilon \mathrm{pF},
\end{aligned}
$$

the maximum error in $\Delta C_{1}$ may be written as

$$
\begin{aligned}
\frac{\delta\left(\Delta C_{1}\right)}{\Delta C_{1}}=(k)+\epsilon\left[\frac{2}{C_{b}}+\frac{3}{C_{1}^{\prime}+C_{b}+C_{c}}\right. & +\frac{3}{C_{1}+C_{b}+C_{c}} \\
& \left.+\frac{2}{C_{1}^{\prime}-C_{1}}\right] .
\end{aligned}
$$

The term involving $\epsilon$ may be large enough to prevent a good determination of $k$ with a single measurement; but this term is random in nature and may be reduced considerably by repeated measurements.

This technique was tested by measuring accuratelyknown (to within $0.01 \%$ ) capacitors with nominal values of 5,13 , and $15 \mathrm{pF}$, in terms of a $1000 \mathrm{pF}$ incremental capacitor connected in series with a nominally $100 \mathrm{pF}$ capacitor and a nominally $82 \mathrm{pF}$ capacitor. The results of the measurements, which appear in table 2, indicate a linear correction of the

\begin{tabular}{|c|c|c|c|c|c|}
\hline \multirow{2}{*}{$\begin{array}{c}\text { Nominal } \\
\text { capaci- } \\
\text { tance }\end{array}$} & \multirow{2}{*}{$\begin{array}{c}\text { Calculated } \\
\text { capaci- } \\
\text { tance }\end{array}$} & \multicolumn{2}{|c|}{ Series measurement } & \multicolumn{2}{|c|}{ Difference $(\%)$} \\
\hline & & $82 \mathrm{pF}$ & $100 \mathrm{pF}$ & $82 \mathrm{pF}$ & $100 \mathrm{pF}$ \\
\hline $5 \mathrm{pF}$ & 5.0753 & 5.0761 & 5.0757 & 0.016 & 0.008 \\
\hline $12 \mathrm{pF}$ & 11.864 & 11.861 & 11.860 & -.027 & -.036 \\
\hline $15 \mathrm{pF}$ & 15.240 & 15.235 & 15.233 & -.033 & -.064 \\
\hline
\end{tabular}
order of -0.01 to -0.03 percent in this particular capacitor.

TABLE 2. Measurement of coaxial line capacitance standards at $3 \mathrm{MHz}$ using a 1000-pF incremental capacitor with 82-pF and 100-pF series capacitors

\subsection{Measurement of Reference Open-Circuit Admit- tance or Short-Circuit Impedance}

Measurements of admittance or impedance will be in error by the uncertainty in the reference opencircuit admittance, or short-circuit impedance. Since this might be the principal source of error in measuring small immittances, it is desirable to measure these reference immittances accurately.

The measurement described in section 3.1. can be rearranged to allow an admittance bridge to measure the admittance of its reference open circuit or an impedance bridge to measure the impedance or its reference short circuit. Equation (35) may be rearranged to read

$$
Y_{x}\left(Y_{1}-Y_{5}\right)=\left(Y_{5}-Y_{2}\right)\left(Y_{3}+Y_{f 3}\right)+\left(Y_{1}-Y_{2}\right) Y_{f 1} .
$$

When $Y_{x}$ is measured directly with the bridge, the quantity observed, $Y_{m}$, is $Y_{x}$ less the admittance removed when $Y_{x}$ is connected to the bridge terminal:

$$
Y_{m}=Y_{x}-Y_{f 3}
$$

Two different admittances may be measured, yielding the two equations

$$
\begin{aligned}
\left(Y_{m 1}+Y_{f 3}\right)\left(Y_{1}-Y_{5}\right)=\left(Y_{5}-Y_{2}\right) & \left(Y_{3}+Y_{f 3}\right) \\
& +\left(Y_{1}-Y_{2}\right) Y_{f 1},
\end{aligned}
$$

and

$$
\begin{aligned}
\left(Y_{m 2}+Y_{f 3}\right)\left(Y_{1}-Y_{5}^{\prime}\right)=\left(Y_{5}^{\prime}-Y_{2}\right) & \left(Y_{3}+Y_{f 3}\right) \\
& +\left(Y_{1}-Y_{2}\right) Y_{f 1},
\end{aligned}
$$

where it is now assumed that the two fringe admittances of the two-port are equal to $Y_{f 1}$. Subtracting (48) from (47) and rearranging yields

$$
Y_{f 3}=\frac{Y_{m 2}\left(Y_{1}-Y_{5}^{\prime}\right)-Y_{m 1}\left(Y_{1}-Y_{5}\right)}{2\left(Y_{5}^{\prime}-Y_{5}\right)}-\frac{Y_{3}}{2},
$$

and $Y_{f 3}$ may be obtained. $Y_{f 1}$ may be obtained by substituting $Y_{f 3}$ into one of the original equations.

When $Y_{f 1}=Y_{f 2}=Y_{f 3}=Y_{f}$, as is the case when the terminals of the two-port and the bridge terminal are identical and have identical environments in the opencircuit condition, $Y_{f}$ may be obtained from the equation

$$
Y_{f}=\frac{Y_{m}\left(Y_{1}-Y_{5}\right)}{2\left(Y_{5}-Y_{2}\right)}-\frac{Y_{3}}{2} .
$$

An exactly analagous situation exists for the measurement of the impedance of the short used as the reference for an impedance bridge. In this case, the equations are

$$
Z_{s}=\frac{Z_{m}\left(Z_{1}-Z_{5}\right)}{2\left(Z_{5}-Z_{2}\right)}-\frac{Z_{3}}{2}
$$

for $Z_{s 1}=Z_{s 2}=Z_{s 3}=Z_{s}$ and

$$
Z_{s 3}=\frac{Z_{m 2}\left(Z_{1}-Z_{5}^{\prime}\right)-Z_{m 1}\left(Z_{1}-Z_{5}\right)}{2\left(Z_{5}^{\prime}-Z_{5}\right)}-\frac{Z_{3}}{2}
$$

for $Z_{s 1}=Z_{s 2} \neq Z_{s 3}$.

This technique was used to measure the fringe capacitance of the NBS 0.750 in, $50-\Omega$ coaxial connector at $1 \mathrm{kHz}$, with a 1 -in long closed cap as the reference condition. The value obtained for the fringe capacitance, assuming $C_{f 1}=C_{f 2}=C_{f 3}$, was $0.2078 \mathrm{pF}$ with an estimated standard deviation of $0.0004 \mathrm{pF}$. This value agrees well with the $0.2070 \mathrm{pF}$ obtained by a measurement involving a calculable line section.

An attempt to measure the impedance of the shorted NBS connector at $100 \mathrm{kHz}$, using an impedance bridge, yielded inconsistent values. The inconsistencies are attributed to the fact that the impedance of the short is 
smaller than that which the bridge is capable of resolving. The resistance of the short was then measured at dc using a Wheatstone resistance bridge. The average of the measured values, attributed primarily to contact resistance, was $0.00016 \Omega$, with the values obtained ranging from 0.00004 to $0.00030 \Omega$.

\section{Conclusions}

The application of precision coaxial connectors to radio frequency immittance measurements makes practical the use of measurement techniques which are usually considered useful only at audio frequencies. In particular, series impedances equipped with precision connectors may be treated as two-port devices and accurately evaluated at a given frequency. Using these two-ports as series impedance transformers makes possible a significant range extension for admittance and impedance measuring instruments. These impedance transformers also make it practical to measure very small admittances in terms of large admittance increments - a technique which has been inverted and used to compare $1000 \mathrm{pF}$ incremental capacitors to low-valued, coaxial line standards of capacitance. In addition, it is possible to use an admit- tance bridge to measure its own fringe capacitance and an impedance bridge to measure the impedance of its reference short-circuit by proper application of series impedance transformers.

\section{References}

[1] Fossum, D. E., Progress Report of IEEE Instrumentation and Measurement Group Technical Subcommittee on Precision Coaxial Connectors, IEEE Trans. Instr. Meas. IM-13, No. 4, 285 (Dec. 1964).

[2] Woods, D., A Coaxial connector system for precision R. F. measurement instrument and standards, Proc. IEE 108-B, Paper No. 3499E, pp. 205-213, (March 1961).

[3] Tang, K. Y., Alternating Current Circuits (International Textbook Co., Scranton, Pa.), pp. 194-202 (1951).

[4] Jones, R. N., and Huntley, L. E., Precision coaxial connectors in lumped parameter immittance measurement, IEEE Trans. Instr. Meas. IM-15, No. 4, 375-380, (Dec. 1966).

[5] Topping, J., Errors of Observation and Their Treatment, London Institute of Physics (1955).

[6] Hartshorn, L., Radio Frequency Measurements by Bridge and Resonance Methods, pp. 219-220 (Wiley, New York, N.Y. Feb. 1940).

[7] Field", R. F., and Sinclair, D. B., A method for determining the residual inductance and resistance of a variable air condenser at radio frequencies, Proc. IRE 24, No. 2, 255-274 (Feb. 1936)

(Paper 74C3\&4-300) 